Research Article

\title{
Graphite Oxide under High Pressure: A Raman Spectroscopic Study
}

\author{
Lianqiang Xu and Li Cheng \\ School of Physics and Information Technology, Ningxia Teachers University, Guyuan, Ningxia 756000, China \\ Correspondence should be addressed to Li Cheng; chengli080910@gmail.com
}

Received 19 March 2013; Accepted 23 May 2013

Academic Editor: Raquel Verdejo

Copyright ( 2013 L. Xu and L. Cheng. This is an open access article distributed under the Creative Commons Attribution License, which permits unrestricted use, distribution, and reproduction in any medium, provided the original work is properly cited.

\begin{abstract}
We present a Raman-based study on the graphite oxide under high pressure. The graphite oxide is stable under hydrostatic high pressure ambient. By carefully fitting the $D, G$, and $D^{\prime}$ bands which are merged at some extent, we successfully retrieve the information of peak position evolution of $\mathrm{G}$ band of graphite oxide under pressure, and it is found that the $\mathrm{G}$ band pressure coefficient is very close to that of graphene, and the pressure-induced peak position shift is reversible upon releasing the pressure. The Ramanbased high pressure investigation indicates that the graphite oxide possesses good mechanical ductility despite the fact that it is oxygenated by functional groups. This study provides useful fundamental understanding of graphite oxide.
\end{abstract}

\section{Introduction}

In recent years, graphene attracted tremendous scientific and engineering interests owing to its unique electronic properties and tunable electronic band structures [1,2]. Meanwhile, graphene's chemical derivatives [3], which are obtained by modifying graphene/graphite structurally or chemically, have been studied extensively because of their giant potential applications [4-6]. To date, most graphene-based practical applications are mainly based on using the graphene-derivative materials, particularly that the graphene oxide (GO) has been already applied successfully in practical applications [7-9]. GO is a flexible material that can exist stably in both structures of powder and transparent film. Though GO is an insulating material, it can be switched to conducting phase by reducing it chemically or physically $[3,10,11]$. Thus it is clearly seen that GO is a multifunctional smart material which can be directly used for various practical applications.

The fundamental understanding of GO's physical and chemical properties has been reported largely in the past few years [3, 12-14], but only very limited studies were published on properties of GO under high pressure. Talyzin et al. discovered that $\mathrm{GO}$ is an unusual auxetic material that presents compressibility anomaly [15]. The volume of GO increases with the pressure up to $1.3-1.5 \mathrm{GPa}$ in the water ambient due to the insertion of water into the interlayer space as revealed by detailed X-ray diffraction (XRD) analysis [15]. In addition to XRD-based study, Raman spectroscopy has been proven as one of the most useful and effective methods for probing the physical properties of carbon-based material at high pressure $[16,17]$, and from viewpoint of instrumental operation, Raman technique is more convenient and safe for probing the basic mechanical properties of carbon-based materials compared to that of XRD. However, despite the fact that GO's mechanical property has been successfully used for practical applications [18], there have been no reported Raman studies on GO at high pressure. Motivated by this, we present the Raman studies on the GO's properties at high pressure in this paper.

\section{Experimental Details}

GO samples prepared by traditional modified Hummer's method were used in the experiment [19]. The microstructure of topography of the GO sample was characterized by JEOL 6700 FEG scanning electron microscope (SEM). The carbon- and oxygen-related chemical species of GO were characterized by XPS using Kratos Axis Ultra DLD (delay line detector) spectrometer equipped with a monochromatic $\mathrm{Al} \mathrm{Ka} \mathrm{X-ray} \mathrm{source}(1486.6 \mathrm{eV})$. The X-ray diffraction (XRD) measurement is carried out by using Bruker D8 diffractometer, with a $\mathrm{Cu} \mathrm{K} \alpha$ radiation $(1.54 \AA)$ in the locked-coupled 


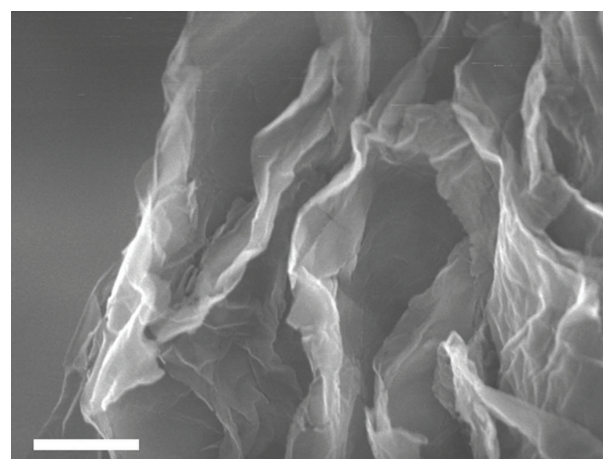

(a)

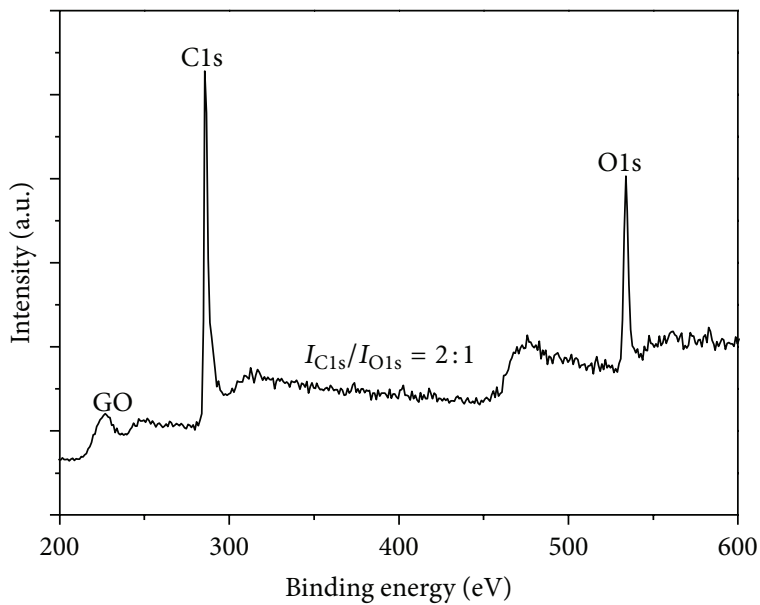

(b)

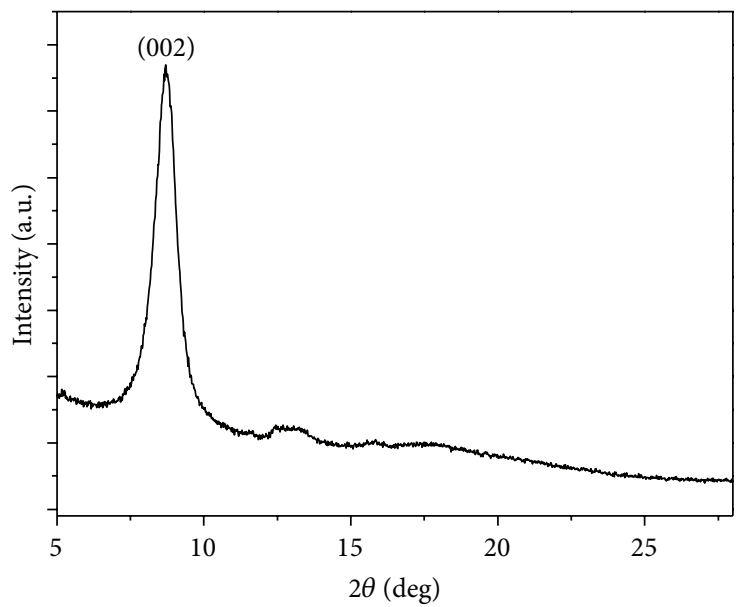

(c)

FIGURE 1: (a) SEM image of GO surface. (b) XPS result of GO. (c) XRD pattern of GO.

geometry of the X-ray gun and detector. The high pressure was generated by a diamond anvil cell, in which the mixture of methanol and ethanol with mole ratio of $4: 1$ is used as a pressure medium. The value of pressure is calibrated by the energy shift of the $R_{1}$ luminescence line of a ruby crystal. The Raman measurement is conducted by Witec CRM200 system with excitation laser wavelength of $532 \mathrm{~nm}$. The laser was focused on the sample by passing through a $50 \mathrm{x}$ objective lens, and the Raman signal is finally collected by the same lens in the backscattering geometry. The laser power is controlled below $0.5 \mathrm{~mW}$ to avoid laser-induced sample heating effect.

\section{Results and Discussion}

From the SEM image shown in Figure 1(a), the GO shows obvious exfoliated structure as compared to that of its precursor graphite powder. The XPS data further confirms that the GO is heavily oxidized as the atomic ratio between $\mathrm{O}$ and $\mathrm{C}$ is $\sim 1: 2$. XRD data shows that the graphite (002) peak at $2 \theta=26.5^{\circ}\left(d_{002}=3.35 \AA\right)$ is fully quenched, and instead, an obvious peak at $2 \theta=8.71^{\circ}\left(d_{002}=10.14 \AA\right)$ is clearly observed. This is the evidence that shows that the interlayer space of graphite is enlarged dramatically after the intercalation of chemical functional groups and water molecules due to the oxidization process $[20,21]$.
Before the high pressure is applied to GO, the typical Raman spectrum measured in ambient air is shown in Figure 2(a). There are two strong active Raman peaks located at $\sim 1350$ and $1600 \mathrm{~cm}^{-1}[3,13]$. The former peak is called D band, which is activated once the defects are introduced into $\mathrm{sp}^{2}$ hybridized carbon networks [22], and the intensity of D band is proportional to the defects concentration [23]. Thus the strong $\mathrm{D}$ band intensity indicates the existence of large amount of defects in $\mathrm{sp}^{2}$-carbon-based materials. The later peak is the $G$ band, arising from the $E_{2 g}$ vibrational mode of $\mathrm{sp}^{2}$-carbon network. It is worth mentioning that the presentation of strong D band at $\sim 1350 \mathrm{~cm}^{-1}$ must accompany the presentation of another defects-assisted Raman band, the $\mathrm{D}^{\prime}$ band, located at $\sim 1620 \mathrm{~cm}^{-1}$. The same as the case of D band, the intensity of $\mathrm{D}^{\prime}$ band is also proportional to the concentration of defects. Furthermore, containing the large amount of defects would give rise to the broadening effect of Raman bands. Thus the normally considered broad "G band" of GO is actually consisting of both $\mathrm{G}$ and $\mathrm{D}^{\prime}$ bands despite the fact that it looks like a single band due to the merge effect after broadening. In addition to the intense $D, G$, and $D^{\prime}$ bands observed obviously, there are three weak Raman active bands at high wavenumber region $\left(>2500 \mathrm{~cm}^{-1}\right)$, which are $2 \mathrm{D}, \mathrm{D}+\mathrm{G}$, and $2 \mathrm{D}^{\prime}$ bands. The $2 \mathrm{D}$ and $2 \mathrm{D}^{\prime}$ bands are secondorder bands of $\mathrm{D}$ and $\mathrm{D}^{\prime}$ bands, respectively. Interestingly, the 


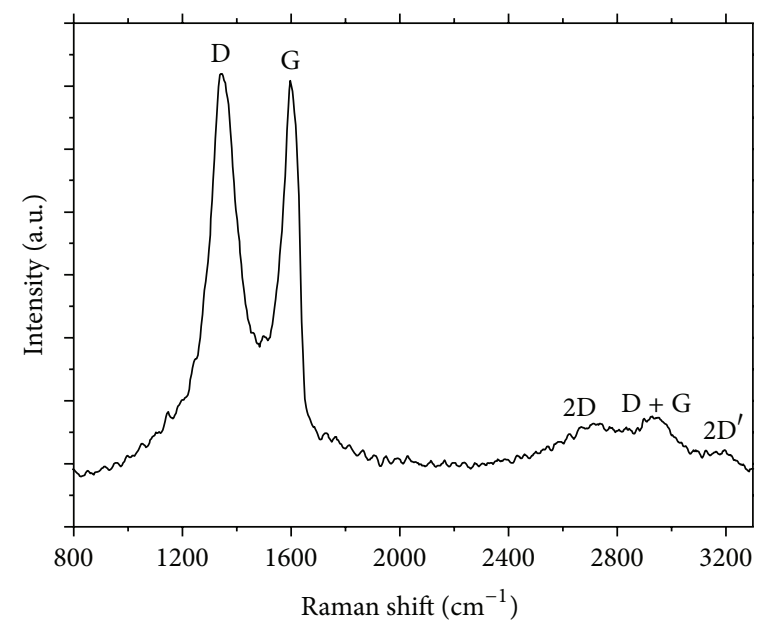

(a)

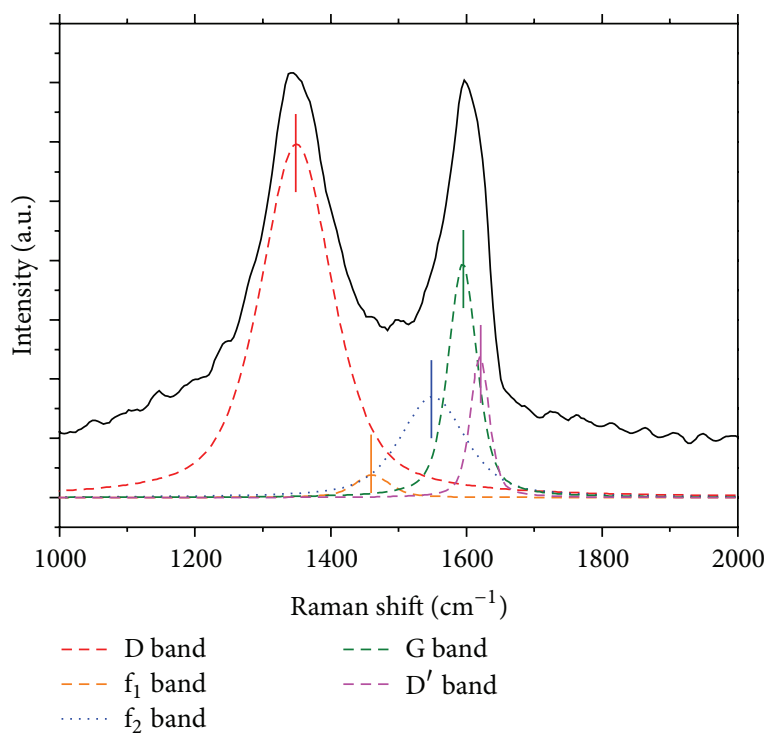

(b)

Figure 2: (a) Raman spectrum of GO excited by $532 \mathrm{~nm}$ laser. (b) The enlarged region of $1000-2000 \mathrm{~cm}^{-1}$, and the five dashed lines are the five deconvoluted Raman peaks.

intensity of both 2D and 2D' bands is inversely proportional to the concentration of defects because both of them are activated by two-phonon (with opposite momentum) assisted double resonant processes. Thus the pristine graphene with perfect $\mathrm{sp}^{2}$ carbon network presents very strong $2 \mathrm{D}$ and $2 \mathrm{D}^{\prime}$ bands, but the intensity decreases dramatically with breaking the $\mathrm{sp}^{2}$-hybridized structure through physical and chemical methods, such as irradiation of plasma/e-beam, oxidization, hydrogenation, and fluorination. The $\mathrm{D}+\mathrm{G}$ band is a combination band which is also reflecting the concentration of defect. It can be obviously observed only when the concentration of defects is high. The shapes of D and $G$ bands do not show obvious change and even the GO is heavily reduced (it is worth noting that the conductivity of reduced GO is normally presenting 4-6 orders higher than those of GO) $[3,10,13]$, but the intensity ratio between $2 \mathrm{D}$ and $\mathrm{D}+\mathrm{G}\left(\mathrm{I}_{2 \mathrm{D}} / \mathrm{I}_{\mathrm{D}+\mathrm{G}}\right)$ bands increases obviously due to the restoration of $\mathrm{sp}^{2}$-hybridized structure [13]. Thus these two Raman active bands are also very important for characterizing GO and RGO. However, for the GO under high pressure, the relatively weak intensities of $2 \mathrm{D}, \mathrm{D}+\mathrm{G}$, and $2 \mathrm{D}^{\prime}$ bands overlap partially with the strong second-order Raman peak from diamond anvils, and hence the peak positions of relatively weak $2 \mathrm{D}, \mathrm{D}+\mathrm{G}$, and $2 \mathrm{D}^{\prime}$ bands are difficult to be exactly determined under pressure. Furthermore, the diamond presents a very strong peak at $\sim 1333 \mathrm{~cm}^{-1}$, which strongly influenced our observation for the peak information of $\mathrm{D}$ band. In our experiment, we will focus on the investigation of the peak position evolution of $G$ and $\mathrm{D}^{\prime}$ bands under high pressure.

For the Raman spectrum of as-received GO, we carefully fitted the peaks in the range between 1000 and $2000 \mathrm{~cm}^{-1}$. In Figure 2(b), the $G$ and $D^{\prime}$ bands are merged together. It is worth noting that, for the highly destroyed $\mathrm{sp}^{2}$-structure, only D, G, and $\mathrm{D}^{\prime}$ bands are not enough to perfectly fit all the Raman signals; thus additional $\mathrm{f}_{1}$ and $\mathrm{f}_{2}$ bands (peak position is between D and G bands as shown in Figure 2(b)) are considered for assisting the fitting result [24]. With introducing the two $\mathrm{f}$ bands, it is clearly seen that the five individual Raman bands of GO at wavenumber range between 1000 and $2000 \mathrm{~cm}^{-1}$ are well fitted (Figure 2(b)).

Although the interlayer space is intercalated by oxygenated functional groups and $\mathrm{H}_{2} \mathrm{O}$, the layered structure of GO still remains [20], which is similar to that of graphite except the fluctuation in layers. Thus the in-plane vibrational $\mathrm{G}$ and $\mathrm{D}^{\prime}$ bands deserved to be independently analyzed. For the Raman spectra of GO sample at pressures, it can be seen in Figure 3 that the Raman intensity ratio between GO (D, G, and $\mathrm{D}^{\prime}$ bands) and diamond $\left(\mathrm{sp}^{3}\right)$ decreases with increasing pressure from 0 to $7.77 \mathrm{GPa}$, and the intensity ratio further decreases when the pressure is released from 7.77 to $0 \mathrm{GPa}$. The peak positions of $\mathrm{G}$ and $\mathrm{D}^{\prime}$ bands for unpressured GO are located at $\sim 1587$ and $\sim 1615 \mathrm{~cm}^{-1}$, respectively. It is worth noting that the peak position of $\mathrm{G}$ band of $\mathrm{GO}$ is slightly larger than pristine graphite/graphene $\left(\sim 1580 \mathrm{~cm}^{-1}\right)$ due to chemical-functional-group-induced hole doping effect. When pressure increases, both $\mathrm{G}$ and $\mathrm{D}^{\prime}$ bands linearly shift to higher wavenumber, and the peak positions blueshift to $\sim 1621$ and $\sim 1649 \mathrm{~cm}^{-1}$ at the maximum pressure $(7.78 \mathrm{GPa})$ for $\mathrm{G}$ and $\mathrm{D}^{\prime}$ bands, respectively. The linearly blueshift of both $\mathrm{G}$ and $\mathrm{D}^{\prime}$ bands indicates that the pressure compresses the $\mathrm{GO}$ which gives rise to the hardening effect of phonons vibration. For the pressure releasing process, the peak positions of both $\mathrm{G}$ and $\mathrm{D}^{\prime}$ bands decrease, and they reach the original values once the pressure is fully released. It indicates that the high pressure (up to $\sim 8 \mathrm{GPa}$ ) induced compression of lattice is reversible. The evolution of peak positions of both $\mathrm{G}$ and $\mathrm{D}^{\prime}$ 


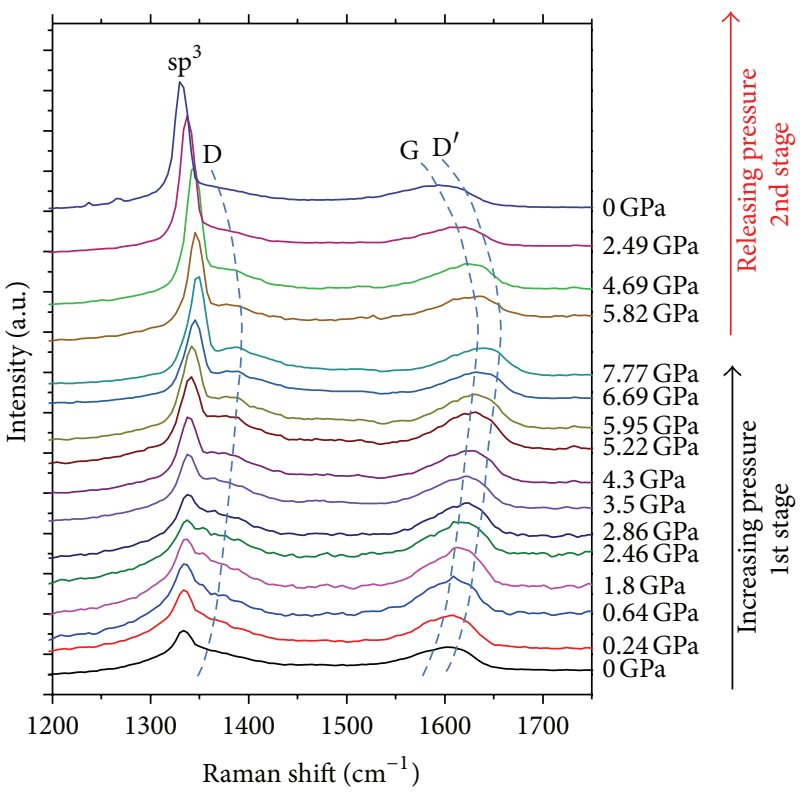

Figure 3: The Raman spectra of GO under various pressures. The first stage shows the evolution of spectra with increasing pressure from 0 to $7.77 \mathrm{GPa}$ gradually, and the second stage shows the evolution of spectra with releasing pressure from 7.77 to $0 \mathrm{GPa}$.

bands with pressure changes can be clearly seen in Figure 4 . There are two phenomena deserved to be mentioned. (a) The slopes of both positions of $\mathrm{G}$ and $\mathrm{D}^{\prime}$ bands vary with pressure are almost the same. (b) The peak positions of both $\mathrm{G}$ and $\mathrm{D}^{\prime}$ bands are reversible upon decreasing the pressure. As shown in Figure 4, the pressure coefficient for $\mathrm{G}_{\text {and }} \mathrm{D}^{\prime}$ bands can be easily calculated, which are $3.8 \mathrm{~cm}^{-1} / \mathrm{GPa}$ for both bands. The pressure coefficient value for G band of GO is smaller than that of both pure graphite $[25,26]$ and pristine grapheme [16]. As the interlayer space of GO is filled out of oxygenated functional groups and water molecules, the adjacent layerlayer coupling is suppressed dramatically due to the enlarged distance (reflected by XRD result as shown in Figure 1(c)), and furthermore, the functional groups (such as hydroxyl, carbonyl, and carboxyl groups) chemically bound to the inplane surface of GO result in the increase of elastic coefficient, which gives rise to the reduction of pressure coefficient. This is also in agreement with the reported result that the effective Young's modulus of single-layer graphene oxide is lower than that of grapheme [27]. It indicates that though the GO is a kind of derivative of graphite with maintained layered structure, its elastic property shows obvious difference compared to that of graphite.

\section{Conclusions}

In summary, Raman spectroscopy has been carried out on $\mathrm{GO}$ under hydrostatic pressure up to $\sim 8 \mathrm{GPa}$. By proper fitting the $\mathrm{G}$ and $\mathrm{D}^{\prime}$ bands of $\mathrm{GO}$ at various pressures, the evolution of pressure-induced $\mathrm{G}$ band shift is clearly revealed, and it is found that the pressure coefficient of G band for GO is smaller than that of graphite and graphene. It is because of

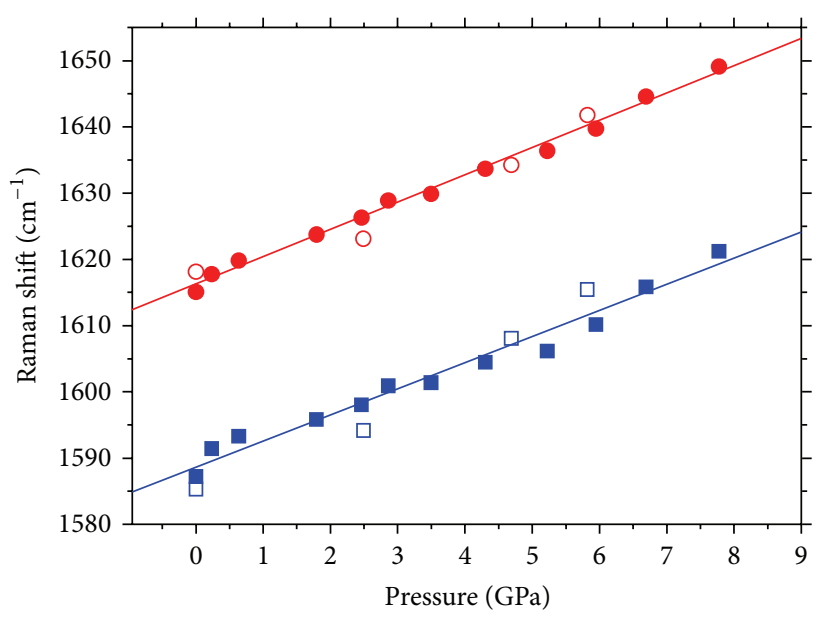

FIGURE 4: The evolution of peak positions of Raman G (blue) and $\mathrm{D}^{\prime}$ (red) bands at high pressures. The solid blue squares and red circles represent the pressure increasing stage, and the opened blue squares and red circles represent the pressure releasing stage.

the fact that the intercalation of both oxygenated functional groups and water molecules suppresses the adjacent layerlayer coupling, and the chemically bonded functional groups at the in-plane surface of GO result in the increase of elastic coefficient. The reversibility of Raman peak positions with respect to the hydrostatic pressure indicates that the GO is a good mechanical ductile material though its elastic property shows some difference compared to graphite. The Raman study of GO's properties at high pressure reveals some fundamental understanding and may be useful for guiding GO-based mechanical applications.

\section{Acknowledgments}

This work was jointly supported by the Natural Science Foundation of Ningxia Hui Autonomous Region of China (Grant no. NZ12227) and the Higher School Scientific Research Foundation of Education Department of Ningxia Hui Autonomous Region of China (Document no. [2011] 263).

\section{References}

[1] Y. Zhang, Y. Tan, H. L. Stormer, and P. Kim, "Experimental observation of the quantum Hall effect and Berry's phase in graphene," Nature, vol. 438, no. 7065, pp. 201-204, 2005.

[2] D. Zhan, J. X. Yan, L. F. Lai, Z. H. Ni, L. Liu, and Z. X. Shen, "Engineering the electronic structure of graphene," Advanced Materials, vol. 24, no. 30, pp. 4055-4069, 2012.

[3] S. Stankovich, D. A. Dikin, R. D. Piner et al., "Synthesis of graphene-based nanosheets via chemical reduction of exfoliated graphite oxide," Carbon, vol. 45, no. 7, pp. 1558-1565, 2007.

[4] L. A. Ponomarenko, F. Schedin, M. I. Katsnelson et al., "Chaotic dirac billiard in graphene quantum dots," Science, vol. 320, no. 5874, pp. 356-358, 2008.

[5] F. Traversi, V. Russo, and R. Sordan, "Integrated complementary graphene inverter," Applied Physics Letters, vol. 94, no. 22, Article ID 223312, 2009. 
[6] G. Eda, G. Fanchini, and M. Chhowalla, "Large-area ultrathin films of reduced graphene oxide as a transparent and flexible electronic material," Nature Nanotechnology, vol. 3, no. 5, pp. 270-274, 2008.

[7] M. D. Stoller, S. Park, Z. Yanwu, J. An, and R. S. Ruoff, "Graphene-Based ultracapacitors," Nano Letters, vol. 8, no. 10, pp. 3498-3502, 2008.

[8] X. Wang, L. Zhi, and K. Müllen, “Transparent, conductive graphene electrodes for dye-sensitized solar cells," Nano Letters, vol. 8, no. 1, pp. 323-327, 2008.

[9] L. F. Lai, L. W. Chen, D. Zhan et al., "One-step synthesis of $\mathrm{NH}$ 2-graphene from in situ graphene-oxide reduction and its improved electrochemical properties," Carbon, vol. 49, no. 10, pp. 3250-3257, 2011.

[10] H. Kang, A. Kulkarni, S. Stankovich, R. S. Ruoff, and S. Baik, "Restoring electrical conductivity of dielectrophoretically assembled graphite oxide sheets by thermal and chemical reduction techniques," Carbon, vol. 47, no. 6, pp. 1520-1525, 2009.

[11] D. Zhan, L. Sun, Z. H. Ni et al., "FeCl3-based few-layer graphene intercalation compounds: single linear dispersion electronic band structure and strong charge transfer doping," Advanced Functional Materials, vol. 20, no. 20, pp. 3504-3509, 2010.

[12] K. A. Mkhoyan, A. W. Contryman, J. Silcox et al., "Atomic and electronic structure of graphene-oxide," Nano Letters, vol. 9, no. 3, pp. 1058-1063, 2009.

[13] D. Zhan, Z. H. Ni, W. Chen et al., "Electronic structure of graphite oxide and thermally reduced graphite oxide," Carbon, vol. 49, no. 4, pp. 1362-1366, 2011.

[14] Y. W. Zhu, S. Murali, W. W. Cai et al., "Graphene and graphene oxide: synthesis, properties, and applications," Advanced Materials, vol. 22, no. 46, p. 5226, 2010.

[15] A. V. Talyzin, V. L. Solozhenko, O. O. Kurakevych et al., "Colossal pressure-induced lattice expansion of graphite oxide in the presence of water," Angewandte Chemie, vol. 47, no. 43, pp. 8268-8271, 2008.

[16] J. E. Proctor, E. Gregoryanz, K. S. Novoselov, M. Lotya, J. N. Coleman, and M. P. Halsall, "High-pressure Raman spectroscopy of graphene," Physical Review B, vol. 80, no. 7, Article ID 073408, 2009.

[17] A. Hadjikhani, J. Chen, S. Das, and W. Choi, "Raman spectroscopy of graphene and plasma treated graphene under high pressure," in Proceedings of the 141st Annual Meeting and Exhibition-Supplemental, Vol 2: Materials Properties, Characterization, and Modeling (Tms '12), pp. 75-79, 2012.

[18] J. T. Robinson, M. Zalalutdinov, J. W. Baldwin et al., "Waferscale reduced graphene oxide films for nanomechanical devices," Nano Letters, vol. 8, no. 10, pp. 3441-3445, 2008.

[19] M. Hirata, T. Gotou, S. Horiuchi, M. Fujiwara, and M. Ohba, "Thin-film particles of graphite oxide 1: high-yield synthesis and flexibility of the particles," Carbon, vol. 42, no. 14, pp. 2929-2937, 2004.

[20] H. K. Jeong, Y. P. Lee, R. J. W. E. Lahaye et al., "Evidence of graphitic $\mathrm{AB}$ stacking order of graphite oxides," Journal of the American Chemical Society, vol. 130, no. 4, pp. 1362-1366, 2008.

[21] A. Lerf, A. Buchsteiner, J. Pieper et al., "Hydration behavior and dynamics of water molecules in graphite oxide," Journal of Physics and Chemistry of Solids, vol. 67, no. 5-6, pp. 1106-1110, 2006.

[22] C. Thomsen and S. Reich, "Double resonant Raman scattering in graphite," Physical Review Letters, vol. 85, no. 24, pp. 52145217, 2000.
[23] L. G. Cançado, A. Jorio, E. H. M. Ferreira et al., "Quantifying defects in graphene via Raman spectroscopy at different excitation energies," Nano Letters, vol. 11, no. 8, pp. 3190-3196, 2011.

[24] S. Mathew, T. K. Chan, D. Zhan et al., "The effect of layer number and substrate on the stability of graphene under $\mathrm{MeV}$ proton beam irradiation," Carbon, vol. 49, no. 5, pp. 1720-1726, 2011.

[25] Z. X. Liu, L. H. Wang, Y. N. Zhao, Q. L. Cui, and G. T. Zou, "High-pressure Raman studies of graphite and ferric chloridegraphite," Journal of Physics, vol. 2, no. 40, pp. 8083-8088, 1990.

[26] M. Hanfland, H. Beister, and K. Syassen, "Graphite under pressure: equation of state and first-order Raman modes," Physical Review B, vol. 39, no. 17, pp. 12598-12603, 1989.

[27] J. W. Suk, R. D. Piner, J. An, and R. S. Ruoff, "Mechanical properties of monolayer graphene oxide," ACS Nano, vol. 4, no. 11, pp. 6557-6564, 2010. 

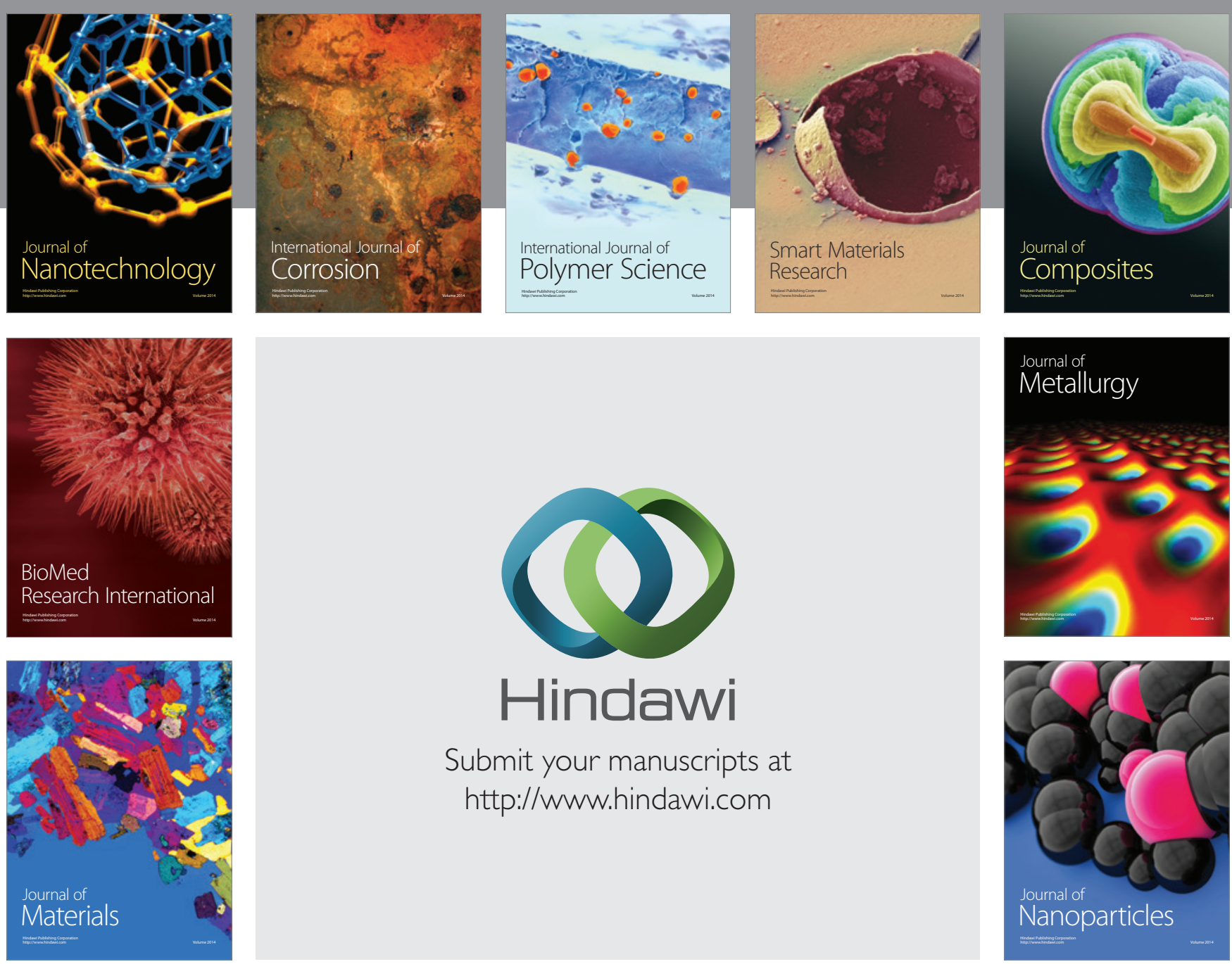

Submit your manuscripts at http://www.hindawi.com
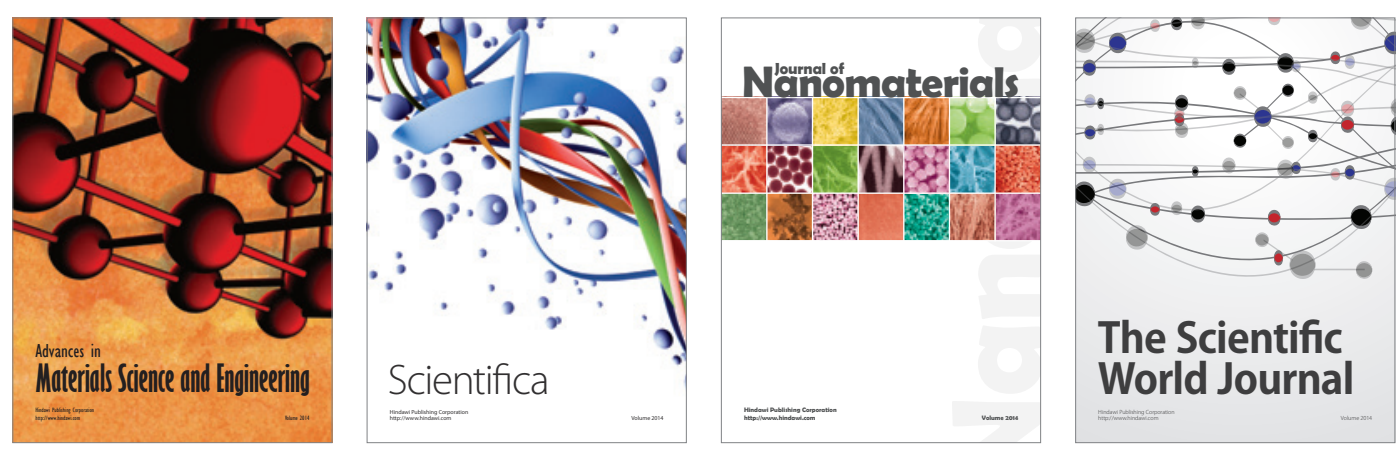

\section{The Scientific World Journal}
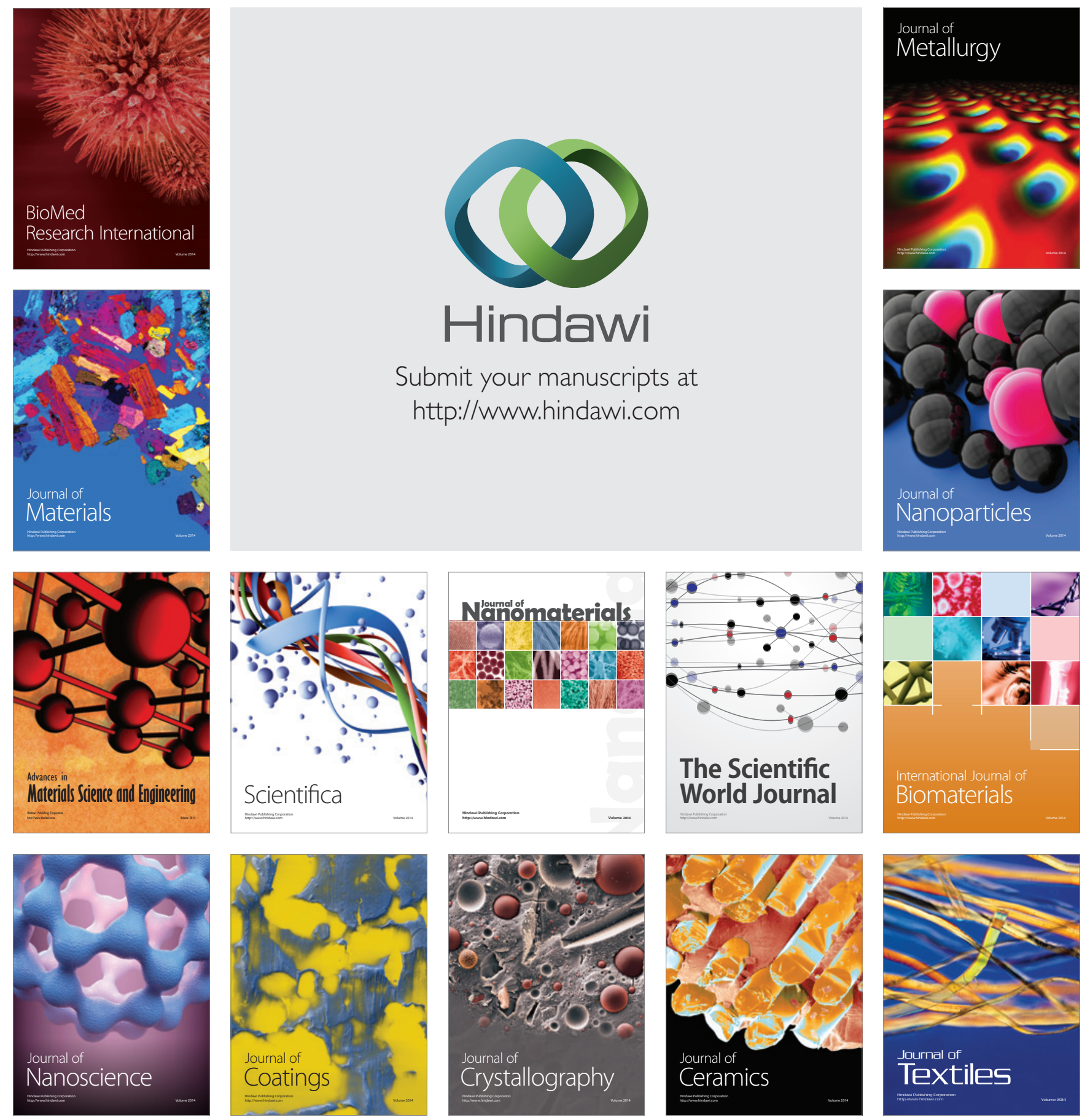